\title{
Application of Rotational Computed Tomography Angiography and 3D Modeling in Planning One-Stage Reconstruction of the Pelvic Floor after Evisceration of the Pelvic Organs
}

\author{
DOI: $10.17691 / \mathrm{stm} 2017.9 .1 .14$
}

Received March 17, 2016

V.S. Ishmetov, MD, DSc, Professor, Head of the Department of X-ray Endovascular Diagnosis and Treatment ${ }^{1}$;

S.I. Blagodarov, Physician in X-ray Endovascular Diagnosis and Treatment';

I.R. Kabirov, PhD Student, Department of Urology2;

A.A. Izmailov, MD, DSc, Professor, Department of Urology2; Head of the Urological Unit';

R.I. Safiullin, MD, DSc, Professor, Department of Urology²;

I.I. Galimov, MD, PhD, Deputy Chief Physician for Surgical Assistance';

R.E. Abdrakhmanov, Physician in X-ray Endovascular Diagnosis and Treatment';

T.R. Ibragimov, Physician in X-ray Endovascular Diagnosis and Treatment';

A.R. Gilemhanov, Physician in X-ray Endovascular Diagnosis and Treatment';

M.F. Urmantsev, MD, PhD, Associate Professor, Department of Urology2; Head of the Oncology Department';

I.F. Sultanov, Anesthesiologist, Department of X-ray Endovascular Diagnosis and Treatment";

I.D. Utenskaya, Anesthesiologist, Department of X-ray Endovascular Diagnosis and Treatment ${ }^{1}$;

V.V. Plechev, MD, DSc, Professor, Head of the Department of Hospital Surgery2;

O.V. Galimov, MD, DSc, Professor, Head of the Department of Surgical Diseases and New Tehnologies

V.N. Pavlov, MD, DSc, Professor, Corresponding Member of the Russian Academy of Sciences,

Head of the Department of Urology, Rector ${ }^{2}$

${ }^{1}$ Bashkir State Medical University Clinic, 2 Shafieva St., Ufa, Bashkortostan, 450083, Russian Federation;

${ }^{2}$ Bashkir State Medical University, 3 Lenin St., Ufa, Bashkortostan, 450008, Russian Federation

The aim of the investigation was to improve perineum plasty method by evaluating the properties of a skin-muscle-fascia flap of the thin thigh muscle (gracilis muscle) using rotational computed tomography angiography and 3D modeling in patients with locally advanced primary or recurrent malignant tumors of the pelvic organs.

Materials and Methods. Transradial access using Innova 3131 IQ angiographic unit (General Electric, USA) was used in the work. A survey dynamic angiography of the abdominal aorta and the lower limb arteries was carried out in order to obtain images of blood vessels and assess the character of blood flow. If atherosclerotic lesions of the iliac or femoral artery segment were detected, stenting or balloon angioplasty was performed.

Results. Rotational computed tomography angiography with 3D modeling in the preoperative period allow the surgeons to obtain information on vascular anatomy of gracilis muscle in patients planned to undergo reconstructive plasty of the perineum by a skin-musclefascial flap of the gracilis muscle. This makes it possible to perform one-stage plastic operation with the best composite flap design selected and to correct the damaged lower extremity arteries without open surgery, if they are detected.

Key words: skin-muscle-fascia flap; evisceration of the pelvic organs; rotational computed tomography angiography; 3D modeling; perineum defects; pelvic organ tumors; reconstructive interventions on the perineum; gracilis muscle.

Complex treatment of the majority of patients with locally extended tumors of the pelvic organs demands vast surgical resection such as evisceration of pelvic organs (EPO). Total evisceration is the removal of all organs of the reproductive system en bloc, which is completed with forming colostomies and urostomies on the anterior abdominal wall (in the exceptional case, orthotopic plasty of the urinary bladder and low rectal anastomosis are performed) [1-4]. EPO remains the best variant of treatment but results in forming a significant anatomic and functional defect of the pelvic floor tissues and perineum the reconstruction of which is a complicated final stage of a long-term operative intervention.

In modern surgical practice, various methods of replacing perineum defects are presented: from closing to reconstructive plastic surgery with application of diverse variants of free and transferred flaps. Application of regionally transferred flaps is one of the most reliable and simplest ways of pelvic floor defect reconstruction.

Skin-muscle flap of the gluteus maximus muscle, skin-muscle-fascia flap of the thin thigh muscle (gracilis

For contacts: Sergey I. Blagodarov, e-mail: blagodarovsi.x-ray@mail.ru 
muscle) and transfer vertical skin-muscle flap of the abdominal rectus muscle are used most commonly [3, 5-7]. Application of the gracilis muscle as a basis of the skin-muscle-fascia transfer flaps with axial blood supply is favored by an easy access for harvesting, the possibility of bilateral plasty, if necessary, and minimal early and late complications at the site of the donor area [4].

However, transfer of the regional flap of the gracilis muscle is limited by the length of the vascular pedicle and small possibilities of changing the size of the cutaneous part of the flap. According to Mathes and Nahai classification (1981), based on the character of blood supply, a flap from the gracilis muscle is referred to type II. This means that blood is supplied to this muscle through one dominant feeding artery and two-three supplementary arteries. The technique of gracilis muscle flap harvesting implies isolation of only main vascularnervous pedicle. Auxiliary sources of arterial inflow are ligated and transacted.

The isolation of the vascular-nervous fascicle is the most difficult stage of flap harvesting, and the length of the basic feeding artery and the variant it originates from the main artery determine in many respects the mobility of the regional flap. Damage of the dominant artery causes impairment of flap blood supply resulting in early complications such as necrosis of the parts or all flap layers. The dominant artery, supplying the gracilis muscle, is known to arise from the profunda femoris artery, the arteries of adductor muscles, or medial femoral circumflex artery [8]. Anatomical variability of axis direction and branching of the main feeding artery of the gracilis muscle create additional technical difficulties for a surgeon team. The complementary manipulations with tissues and vessels performed in such cases, the larger extent of tissue dissections and longer time of the operation influence negatively the results of the pelvic floor plastic surgery. It should be also noted, that even technically ideally performed flap harvesting is accompanied by transitional blood supply disturbances, especially in the cutaneous part [7-9]. The morphometric characteristics of the gracilis muscle, described in the literature, such as muscle belly size, length, diameter and the variant of the main feeding artery branching from the great vessel are population-based and cannot be definitely used for a concrete patient of a certain age and gender. This is due to a marked individual variability of vessels, their quantity, localization of the axis, and the point of entry into the gracilis muscle. Even comparing the contralateral segments of one and the same individual this variability can be frequently found [10]. Besides, comorbidities are often found in patients in the course of their examination. Pathology of the cardiovascular system and diabetes mellitus are revealed most commonly, the ailments which slow down the blood flow velocity in the great arteries and reduce the indices microcirculation of tissues composing the flap. That is why it is necessary to evaluate clearly the situation with the vessels in the gracilis muscle in order to improve the results of one-stage reconstruction of the pelvic floor after evisceration of the pelvic organs. Such assessment can be done with the help of rotational computed tomography angiography (RCTA) and 3D modeling.

The aim of the investigation was to improve perineum plasty method by evaluating the properties of a skin-muscle-fascia flap of the gracilis muscle using rotational computed tomography angiography and 3D modeling in patients with locally advanced primary or recurrent malignant tumors of the pelvic organs.

Materials and Methods. The study was carried out in the Clinic of Bashkir State Medical University using transradial access on Innova 3131 IQ angiographic unit (General Electric, USA). The study complies with the Declaration of Helsinki (the Declaration was passed in June 1964, Helsinki, Finland, and revised in October 2000, Edinburg, Scotland) and was performed following approval by the Ethic Committee of Bashkir State Medical University Clinic. Written informed consent was obtained from every patient.

A survey dynamic angiography of the abdominal part of the aorta and lower limb arteries. At the first stage, a survey dynamic angiography of the abdominal part of the aorta and lower limb arteries was performed in order to obtain the images of blood vessels and evaluate the character of blood flow (Figure 1). If atherosclerotic lesions of the iliac or femoral artery segment were detected, stenting or balloon angioplasty was performed.

In the period from 2008 to 2015, 36 EPO have been performed, of them total supralevator eviscerations was done in 26 cases, and infralevator evisceration in

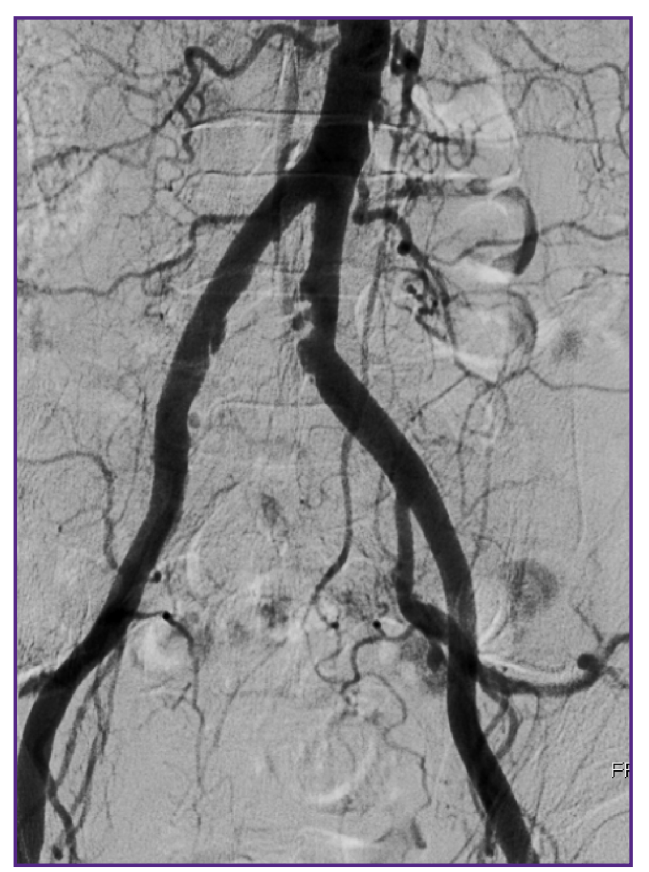

Figure 1. Survey transradial dynamic angiography of the abdominal part of the aorta and lower limb arteries 


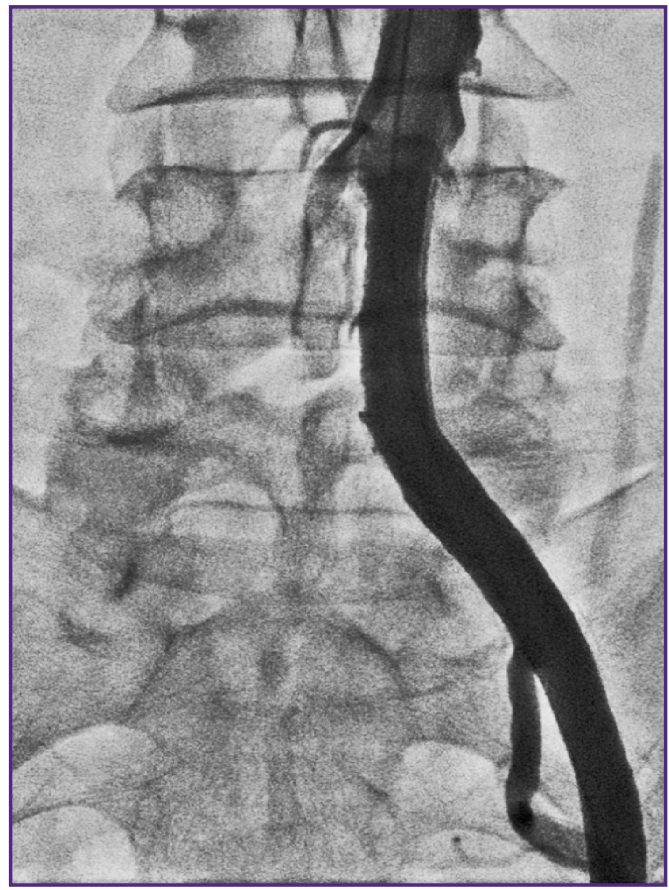

Figure 2. The result of the left common iliac artery stenting

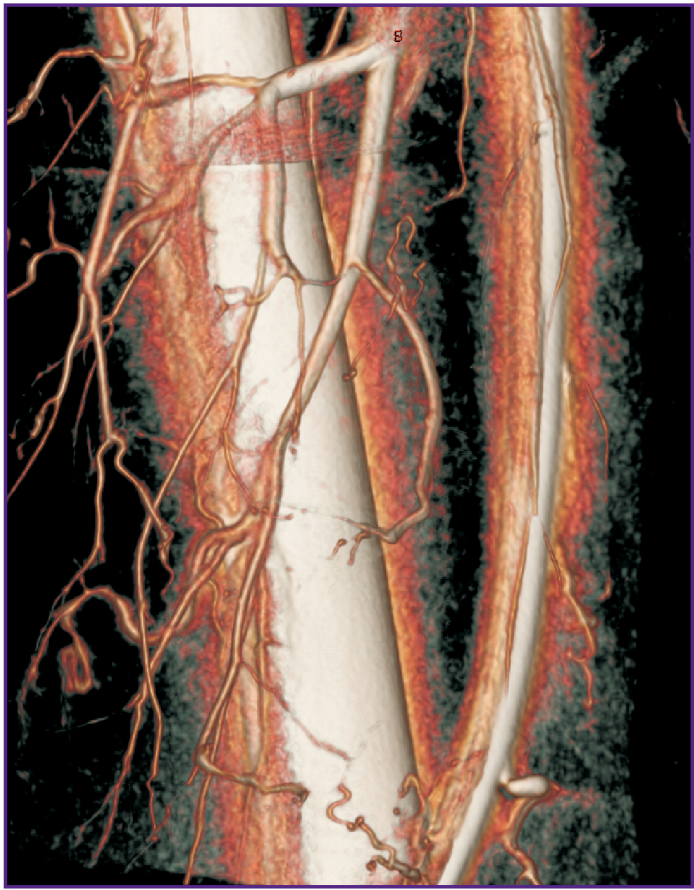

Figure 3. 3D reconstruction of the lower limb vascular anatomy after rotational computed tomography angiography
10 cases with plastic surgery of the pelvic floor by a skinmuscle-fascia flap of the gracilis muscle in both groups. All patients underwent RCTA as a standard examination before the operative intervention.

Significant atherosclerotic damage of the iliac segment arteries was found in 6 cases of group 1 and in 4 cases of group 2. In all these cases stenting was performed using Abbott Absolute Pro and Abbott Omnilink Elite stents (Abbott Vascular, USA) (Figure 2).

Selective rotational computed tomography angiography. At the second stage, selective RCTA was carried out. The procedure took $20 \mathrm{~min}$ per patient on average, and in case of stenting $40 \mathrm{~min}$. The total amount of the contrast material did not exceed $100.0 \mathrm{ml}$, and for cases with stents $200.0 \mathrm{ml}$.

Assessment and conversion of the obtained data. The data obtained were analyzed on the Advantage Workstation VolumeShare 4 (GE Healthcare, USA) using special algorithms for image reconstruction (Figure 3).

The best projection for visualization of the artery feeding the gracilis muscle was determined by transformation of the obtained images in the modes of multiplanar reconstruction and maximal intensity projection.

Preliminary assessment of the 3D vessel images after data reconstruction was carried out with a more precise definition of morphometric characteristics of the femoral gracilis muscle and lumen diameters of the great vessels and their branches. Using these 3D vessel images the origins of the arteries supplying blood to the gracilis muscle were identified, and their length measured, which helped determine the extent of flap advancement and rotation.

We analyzed the rate and character of the common surgical complications associated with various methods of EPO, and evaluated the viability of the plastic material employed in perineum plasty, functional state of the operated zone, and the esthetic result.

Results and Discussion. RCTA with 3D modeling in the preoperative period allowed us to obtain information about the vascular anatomy of the gracilis muscle in 36 patients who were planned to undergo reconstructive plastic surgery of the perineum with a skin-muscle-fascia flap of the gracilis muscle.

Arteries feeding the gracilis muscle were visualized in all 36 patients, and in 10 cases a supplementary correction of blood flow in the lower limb arteries was performed to prevent early postoperative complications after one-stage reconstruction of the pelvic floor following EPO.

Most commonly the dominant artery originated from the profunda femoris artery (28 patients, $77 \%$ ) and femoral circumflex artery (8 patients, $23 \%)$. The results of the gracilis muscle morphometric investigation and the analysis of the anatomic structure variants of the profunda femoris artery branches, as well as the anatomic characteristics of the gracilis muscle are presented in the Table. In all cases of preoperative planning of a flap harvesting on the basis of the gracilis muscle, RCTA with 3D modeling enabled precise visualization of the zone where the main feeding artery enters the gracilis muscle and integumentary tissues. 
Average parameter values of the planned flap on the basis of the gracilis muscle

\begin{tabular}{lcc}
\hline \multicolumn{1}{c}{ Parameter } & Mean value $(\mathrm{mm})$ & Range \\
\hline Muscle length $(\mathrm{mm})$ & 305.9 & $245.0-350.0$ \\
\hline Length of the main feeding artery $(\mathrm{mm})$ & 76.0 & $56.0-114.0$ \\
\hline Artery diameter in the proximal part $(\mathrm{mm})$ & 2.5 & $0.9-5.0$ \\
\hline Artery diameter in the distal part at the level of entering the muscle belly $(\mathrm{mm})$ & 1.1 & $0.9-1.9$ \\
\hline Distance from the main artery to the pubic bone $(\mathrm{mm})$ & 82.3 & $78.0-114.0$ \\
\hline Distance from the main artery to the proximal supplementary artery $(\mathrm{mm})$ & 36 & $32.0-40.0$ \\
\hline Quantity of supplementary arteries & 2.5 & $2.0-3.0$ \\
\hline $\begin{array}{l}\text { Distance from the supplementary artery to the pubic bone }(\mathrm{mm}): \\
\quad \text { 1 }^{\text {st }} \text { supplementary artery } \\
2^{\text {nd }} \text { supplementary artery } \\
3^{\text {rd }} \text { supplementary artery }\end{array}$ & 16.5 & $14.2-17.8$ \\
\hline
\end{tabular}

In all cases the arteries feeding the gracilis muscle were determined to be suitable for the reconstructive plastic surgery of the perineum. It should be noted that in case of abnormal branching of the dominant artery, there always existed the possibility of using contralateral segment for flap formation.

Infralevator EPO operations with the restoration of the pelvic floor lasted $285 \mathrm{~min}$, on average, the mean blood loss volume amounted to $595 \mathrm{ml}$. The same average parameters for supralevator EPO were $270 \mathrm{~min}$ and $420 \mathrm{ml}$, respectively. These values in the tested group did not differ significantly from the parameters in the group of patients undergone EPO without pelvic floor reconstruction.

In the postoperative period necrosis of the epidermal part of the flap was diagnosed in 1 patient from the group of infralevator EPO with pelvic floor reconstruction. In this case it was decided to perform plastic operation with a skin-fascia flap. Necrosis of the epidermal part of the flap was thought to be caused by disturbances of skin microcirculation, since the viability of the deep flap layers remained intact.

At present, the reconstructive perineum plasty with a skin-muscle-fascia flap harvested from the gracilis muscle is maximally adapted to oncological practice. Close position of the donor area to the perineum offers a good possibility to replace the pelvic floor defect by a gracilis muscle flap after EPO. Harvesting a gracilis muscle flap presents no functional problems for lower extremities, does not weaken the state of the patient anterior abdominal wall and does not create any difficulties connected with bringing urostomies and colostomies out on the anterior abdominal wall.

In each individual case, the design of the composite flap on the basis of the gracilis muscle may be defined individually depending on the process extension, findings of the examination conducted, composition and character of the planned tissue defect, and somatic condition of the patient. The instrumental investigations being performed are sufficient for determining flap design, visualization and assessment of the artery in the feeding pedicle of the planned flap. Visualization of the vascular landmarks during complicated flap formation significantly improves isolation of the flap vascular pedicle up to its origin from the great artery, reducing the operating time.

Thus, to know specific features of architectonics of the branches in the gracilis muscle area is of great importance for performing reconstructive plastic surgery of the perineum using a gracilis muscle flap. The presented method of defining the variants of gracilis muscle blood supply is rather simple and efficacious enough, does not rise the cost of treatment and does not increase the radiation dose to patients. This enables the definition of the specificity of the lower limb vascular anatomy, and, if the damaged arteries are detected, their correction without open operative intervention.

Knowledge of blood supply structure allows the surgeon to plan the operation more thoroughly reducing thereby intraoperative and postoperative complications in the perineum and donor zone associated with identification of vessels and determination of viability of the structurally complex flap.

Conclusion. Rotational computed tomography angiography of the lower extremities with 3D modeling is recommended to be included in the complex of examining patients with locally extended tumors of pelvic organs and the necessity of evisceration, when the reconstructive plastic surgery is planned.

Study Funding. The work was not supported by any source.

Conflicts of Interest. The authors declare no conflicts of interest related to this study.

\section{References}

1. Khinman F. Operativnaya urologiya: atlas [Operative urology: atlas]. Pod red. Yu.G. Alyaeva, V.A. Grigoryana 
[Yu.G. Alyaev, V.A. Grigoryan (editors)]. Moscow: GEOTARMedia; 2007; 1192 p.

2. Wechselberger G., Schubert H.M., Schoeller T. Der freie Musculus-gracilis-Lappen zur Weichteildefektdeckung. Oper Orthop Traumatol 2008; 20(2): 119-127, https://doi. org/10.1007/s00064-008-1235-9.

3. Aglullin I.R., Didakunan F.I., Ziganshin M.I., Valiev A.A., Aglullin T.I., Safin I.R., Aglullin M.I. Technical aspects of evisceration pelvis organs. Povolzhskiy onkologicheskiy vestnik 2015; 4: 63-69.

4. Davydov M.I., Odaryuk T.S., Nechushkin M.I., Fainshtein I.A., Trigolosov A.V., Strakhov V.Yu., Gevorkyan T.G. Surgical treatment policy for locally advanced small pelvic tumors involving the bladder. Onkourologiya 2006; 2: 26-30.

5. Bullard K.M., Trudel J.L., Baxter N.N., Rothenberger D.A. Primary perineal wound closure after preoperative radiotherapy and abdominoperineal resection has a high incidence of wound failure. Dis Colon Rectum 2005; 48(3): 438-443, https://doi.org/10.1007/s10350-0040827-1.

6. Nisar P.J., Scott H.J. Myocutaneous flap reconstruction of the pelvis after abdominoperineal excision. Colorectal
Dis 2009; 11(8): 806-816, https://doi.org/10.1111/j.14631318.2008.01743.x.

7. Chan S., Miller M., Ng R., Ross D., Roblin P., Carapeti E., Williams A.B., George M.L. Use of myocutaneous flaps for perineal closure following abdominoperineal excision of the rectum for adenocarcinoma. Colorectal Dis 2010; 12(6): 555-560, https://doi.org/10.1111/j.1463-1318.2009.01844.x.

8. Kind G.M., Foster R.D. The longitudinal gracilis myocutaneous flap: broadening options in breast reconstruction. Ann Plast Surg 2008; 61(5): 513-520, https:// doi.org/10.1097/sap.0b013e318168db64.

9. Macchi V., Vigato E., Porzionato A., Tiengo C., Stecco C., Parenti A., Morra A., Bassetto F., Mazzoleni F., De Caro R. The gracilis muscle and its use in clinical reconstruction: an anatomical, embryological, and radiological study. Clin Anat 2008; 21(7): 696-704, https://doi.org/10.1002/ca.20685.

10. Chadwick M.A., Vieten D., Pettitt E., Dixon A.R., Roe A.M. Short course preoperative radiotherapy is the single most important risk factor for perineal wound complications after abdominoperineal excision of the rectum. Colorectal Dis 2006; 8(9): 756-761, https://doi.org/10.1111/j.14631318.2006.01029.x. 